\title{
Isolierte Olekranonfrakturen des Erwachsenen
}

\author{
Manuel Baacke, Atesch Ateschrang, Martin Lucke, Ulrich Stöckle
}

\section{Zusammenfassung}

Ellengelenksfrakturen sind mit 7\% aller Frakturen eher selten. 38\% davon betreffen isoliert das Olekranon. Ursache ist meist ein direktes Trauma mit Sturz auf den flektierten Ellenbogen. Haupttherapieziele sind die Herstellung der Gelenkstabilität, die möglichst stufenfreie anatomische Gelenkrekonstruktion und eine frühfunktionelle Übungsstabilität. Daher besteht in den meisten Fällen eine Operationsindikation. Die etabliertesten und am besten nachuntersuchten Verfahren stellen die Zuggurtungs-/Plattenosteosynthesen dar. Die Wahl des Osteosyntheseverfahrens richtet sich dabei v.a. nach der Frakturmorphologie. Für Fälle mit ausgedehnten Trümmerzonen und osteoporotischen Knochenverhältnissen stehen mittlerweile anatomisch vorgeformte winkelstabile Implantate zur Verfügung. Diese ermöglichen aufgrund mehrerer winkelstabiler Schraubenoptionen im gelenkflächentragenden Anteil ein breites Einsatzspektrum und Primärstabilität.

\section{Isolated Fractures of the Olecranon in Adults}

Fractures of the elbow joint are rather rare amounting to $7 \%$ of all fractures. Of all elbow fractures 38\% are isolated olecranon fractures. The most common cause is a direct trauma on the flexed elbow. The main treatment goals are joint stability, an anatomic reconstruction of the joint surface and the possibility of an early restoration of functionality. Therefore the need for an operation exists in most cases. The most established, commonly used and best reviewed procedures include the tension band wiring and the plate osteosynthesis. The choice of the technique depends mainly on the morphology of the fracture. For patients with multifragmentary fractures and osteoporosis, anatomically preshaped angular stable implants are meanwhile available. These plates enable a wide operational range and primary stability based on several angular stable screw options in the fragment forming the joint surface.

\section{Epidemiologie und Unfallmechanismus}

Insgesamt sind Frakturen des Ellengelenks mit etwa 7\% aller Brüche des Erwachsenen relativ selten. Sie weisen 2 Häufigkeitsgipfel auf, um das 20. und das 65. Lebensjahr. In 38\% der Fälle ist das Olekranon isoliert betroffen. Ursächlich für Olekranonfrakturen ist meist das direkte Trauma, der Sturz auf den gebeugten Ellenbogen. Durch diesen Mechanismus entstehen vorwiegend Querfrakturen. Durch die axiale Kraft-

OP-JOURNAL 2012; 28: 20-26

(c) Georg Thieme Verlag KG Stuttgart · New York DOI http://dx.doi.org/10.1055/s-0032-1314967 einleitung, das Einstauchen der Trochlea humeri erklären sich die häufigen Mehrfragmentfrakturen mit Impression der Gelenkfläche. Indirekt kann es beim Sturz auf den gestreckten Unterarm durch einen Hebelmechanismus an der Trochlea humeri und Trizepsanspannung zu Schrägfrakturen im Sinne einer Avulsionsverletzung kommen.

Zur Dislokation der Fragmente kommt es durch den Zug des M. triceps brachii [1].

\section{Diagnostik}

Diagnostisch sind die Rekonstruktion des Unfallmechanismus sowie das klinische Bild wegweisend. Inspektorisch zeigen sich meist eine leicht gestreckte Schonhaltung des betroffenen Armes, lokale Weichteilverletzungen/-schwellungen und ggf. Asymmetrien des Hueter'schen Dreiecks (gleichschenkliges Dreieck zwischen Epicondylus humeri radialis/ulnaris, Olekranonspitze). Palpatorisch kann sich als Folge eines dislozierten proximalen Fragments ein subkutaner knöcherner Defekt finden. Funktionell besteht eine schmerzhaft eingeschränkte oder aufgehobene aktive und passive Ellenbogenbeweglichkeit.

Obligat ist eine Prüfung v. a. des N. ulnaris, welcher aufgrund seiner topografischen Nähe geschädigt werden kann.

An Bildgebung ist die konventionelle Röntgenuntersuchung des Ellenbogens in 2 Ebenen in aller Regel ausreichend. Die zusätzliche Computertomografie hat v.a. bei Mehrfragmentfrakturen und komplexen knöchernen Begleitverletzungen zur präoperativen Planung Bedeutung [2].

\section{Klassifikation}

Nach der AO (Arbeitsgemeinschaft Osteosynthese) werden die Frakturen des Olekranons den proximalen Unterarmfrakturen zugeteilt (Abb.1). In der klinischen Anwendung ist die Mayo-Klassifikation sehr gebräuchlich (Abb. 2). Diese Einteilung beruht neben der Frakturmorphologie auf dem Grad der Frakturdislokation. Zudem werden Subluxationsstellungen im Humeroulnargelenk als Ausdruck einer Gelenkinstabilität berücksichtigt.

\section{Therapie}

Vorrangiges Ziel der Behandlung von Frakturen des Olekranons sind die anatomisch stufenfreie Rekonstruktion der Gelenkfläche und die Gelenkstabilisierung. 


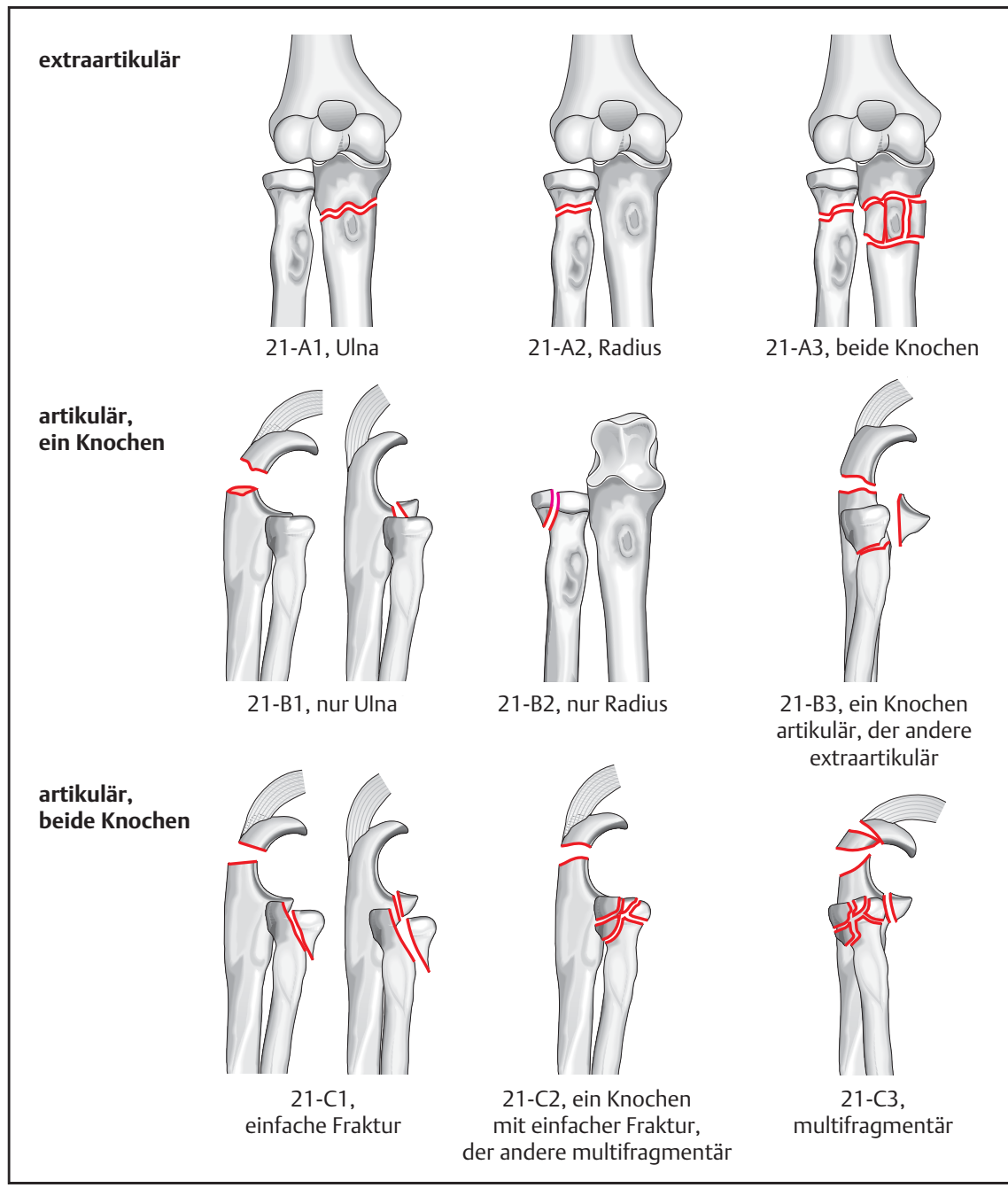

Abb. 1 AO-Klassifikation der Frakturen von proximalem Radius und proximaler Ulna. (Aus: Stöckle U, Hrsg. Ellenbogenchirurgie Standardverfahren, Tipps und Tricks. 2010. Mit freundlicher Genehmigung des Elsevier-Verlages, München.)

Die Behandlung soll v.a. eine frühfunktionelle krankengymnastische Beübung des verletzten Gelenks ermöglichen, um einer posttraumatischen Einsteifung entgegenzuwirken. Da es sich bei Olekranonfrakturen aufgrund von Unfallmechanismus und Trizepssehnenzug überwiegend um dislozierte Frakturen handelt, werden die meisten Frakturen einer operativen Therapie zugeführt. Die Therapieentscheidung richtet sich allerdings auch nach Alter, Aktivitätsniveau und Komorbiditäten der Betroffenen.

\section{Konservative Therapie}

In der Literatur wird, ein stabiles Ellenbogengelenk vorausgesetzt, die konservative Behandlung von Olekranonfrakturen bei einer Fragmentdislokation von bis zu $2 \mathrm{~mm}$ akzeptiert. Es erfolgt die Ruhigstellung in einer dorsalen Oberarmschiene in leichter Streckstellung. Nach einer Woche wird eine Röntgenkontrolle durchgeführt, um eine sekundäre Dislokation sicher ausschließen zu können. Anschließend beginnt die krankengymnastische Beübung aus der Schiene heraus mit geführten Bewegungen. Die Flexion bleibt für mindestens 3 Wochen nach dem Unfallereignis auf maximal $90^{\circ}$ limitiert, Umwendbewegungen sind schmerzadaptiert frei zu beüben. Engmaschige klinisch-radiologische Kontrollen stellen eine Voraussetzung für die konservative Behandlung dar.

\section{Operative Therapie}

\section{Präoperative Planung}

Die Wahl des Osteosyntheseverfahrens wird überwiegend von der Frakturmorphologie bestimmt. Begleitende Weichteilverletzungen beeinflussen eher den Zeitpunkt der operativen Versorgung. Offene Frakturen müssen notfallmäßig operativ behandelt werden. Je nach Grad der Weichteilschädigung kann eine einzeitige definitive osteosynthetische Versorgung bzw. muss eine zweizeitige Versorgung mittels temporär angelegtem ellengelenksüberbrückenden Fixateur externe bis zur Weichteilkonsolidierung durchgeführt werden. Da Olekranonfrakturen meist mit Hautschürfungen und -kontusionen im Zugangsbereich assoziiert sind, sollte eine möglichst zeitnahe osteosynthetische Versorgung erfolgen. Außerdem wirkt sich eine verlängerte präoperative Ruhigstellung hinsichtlich der Funktion bei der frühfunktionellen Beübung nachteilig aus [3].

Die etabliertesten und am besten nachuntersuchten Osteosyntheseformen sind die Zuggurtungs- und die Plattenosteosynthese. Beide Verfahren sollen im Folgenden näher erläutert werden. Die Operation kann in Rückenlage mit über dem Bauch gelagertem Arm, in Seit- oder Bauchlage zur einfacheren Exposition durchgeführt werden.

\section{Zuggurtungsosteosynthese}

Bei der Zuggurtungsosteosynthese werden die Zugkräfte der Trizepssehne zu Kompressionskräften im Frakturbereich umgewandelt.

Dieses Prinzip hat v.a. dort Bedeutung, wo Frakturform und Knochenqualität eine stabile Abstützung der Fragmente unter Kompression zulassen, z. B. bei einfachen, quer verlaufenden Frakturen. Weniger geeignet ist die Methode bei Schräg- oder Trümmerfrakturen, bzw. bei Frakturen distal des Rotationszentrums des Olekranons.

Mit einem Einzinkerhaken oder einer spitzen Repositionszange erfolgt die Sicherung und temporäre Fixation der reponierten Fraktur. Um einen sicheren Halt der Zange am Schaft zu erreichen, kann ein monokortikales Loch in den frakturnahen Kortex des Schaftes gebohrt werden, in welchem sich die schaftseitige Zangenbranche verankern lässt. Nach Bildwandler-Dokumentation eines exakten Repositionsergebnisses werden 2 Kirschner-Drähte der Stärke 1,6-2 mm unter Verwendung einer Parallelbohrbüchse von proximal eingebracht (Abb.3a). Die Drähte sollten einen parallelen Verlauf relativ dicht unterhalb der Gelenkfläche aufweisen und mit ihren Spitzen die Gegenkortikalis gerade durchbohrt haben. Auf einen ausreichenden Abstand der Drähte zueinander ist zu achten, da hierdurch die Rota- 
tionsstabilität der Osteosynthese erhöht wird. Nach Perforation der Gegenkortikalis mit den Kirschner-Drähten werden diese um ca. $1 \mathrm{~cm}$ zurückgezogen und anschließend gekürzt, sodass sie den Knochen proximal um ca. $2 \mathrm{~cm}$ überragen (Abb.3b). Schräg abgekniffene Drahtenden erleichtern später - nach entsprechendem Umbiegen - das Versenken im Knochen. Etwa $4 \mathrm{~cm}$ distal der Fraktur wird in einem Abstand von ca. $5 \mathrm{~mm}$ ventral der dorsalen Kortikalis die Ulna mit einem 2-mm-Bohrer quer durchbohrt. Durch dieses Loch wird ein Cerclagendraht der Stärke $1,25 \mathrm{~mm}$ geführt. Auf der einen Seite kann ein „Auge“ in den Draht eingedreht werden, welches später beim Anspannen der Cerclage als Ausgang für den entsprechenden „Zwirbel“ dient (Abb. 3 b). Anschließend wird der Draht knochennah durch den Sehnenansatz in einer Achtertour um die Kirschner-Drähte geführt. Der Draht ist so auszurichten, dass die beiden Enden zu einem „Zwirbel“ verquirlt werden können, der dem zuvor eingearbeiteten „Auge“ gegenüber auf dem anderen Schenkel der Cerclage liegt. Nun können beide Cerclageschenkel simultan mit 2 Zangen ausgewogen verspannt werden (Abb.3c und d). Die Drahtzwirbel werden ggf. gekürzt, mit einer Spitzzange umgebogen und dem Knochen angelegt, um spätere Weichteilirritationen zu vermeiden. Abschließend werden die Enden der Kirschner-Drähte um $180^{\circ}$ umgebogen und mit einem Stößel vorgetrieben. Die Drahtspitzen finden hierdurch wieder ihre wichtige Verankerung in der Gegenkortikalis, ohne diese wesentlich $\mathrm{zu}$ überragen. Gleichzeitig werden zur Weichteilschonung die proximalen Anteile der Kirschner-Drähte tief in den Fasern des Trizepssehnenansatzes versenkt (Abb. 3c, d und 4). Optimalerweise sollte der Cerclagedraht dem Knochen innerhalb der umgebogenen Kirschner-Drähte dicht anliegen.

\section{Plattenosteosynthese}

Als Implantate werden in der Regel eine 3,5-mm-LCDCP bzw. eine KleinfragmentLCP verwendet. Die Plattenlänge richtet sich nach der Frakturlokalisation und deren Ausdehnung.

Nach operativer Frakturdarstellung erfolgt die Reposition analog zur Zuggurtungsosteosynthese. Zunächst müssen jedoch evtl. gelenkflächentragende Zwischenfragmente anatomisch eingepasst werden. Hierbei kann ein temporäres
Typ I

Fraktur nicht disloziert

Ellenbogengelenk stabil

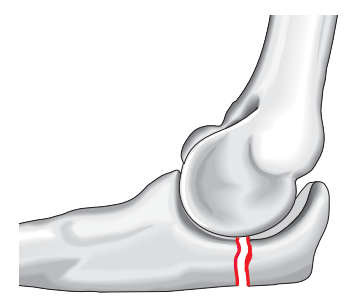

IA einfach

\section{Typ II}

Fraktur disloziert

Ellenbogengelenk stabil

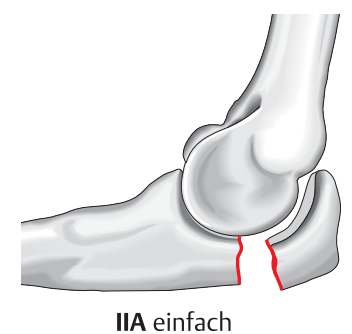

\section{Typ III}

Fraktur disloziert

Ellenbogengelenk instabil

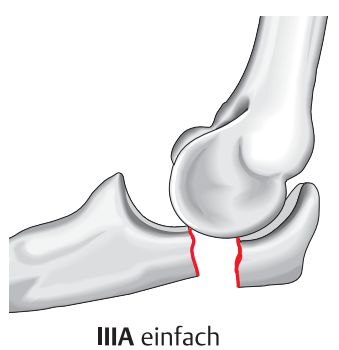

IB mehrfragmentär

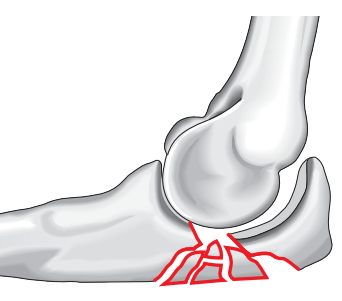

IIB mehrfragmentär

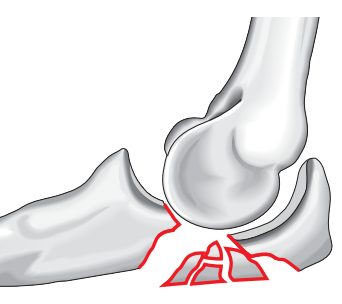

IIIB mehrfragmentär

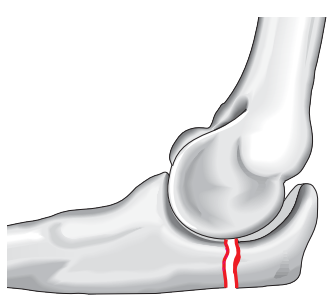

Abb. 2 Mayo-Klassifikation der Olekranonfrakturen. (Aus: Stöckle U, Hrsg. Ellenbogenchirurgie Standardverfahren, Tipps und Tricks. 2010. Mit freundlicher Genehmigung des Elsevier-Verlages, München.)

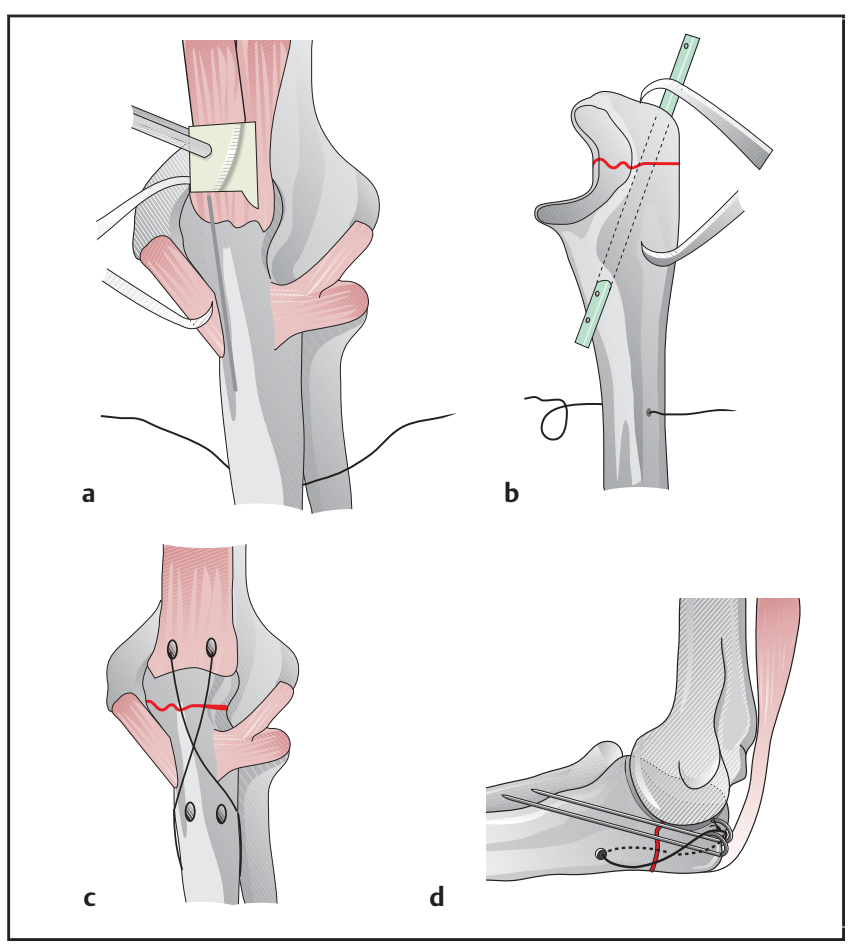

Abb. 3 a bis $\mathbf{d}$

Technik der Zuggurtungsosteosynthese in Anlehnung an die Beschreibung der AO (www.aosurgery.org). a Sicherung der Reposition mit einer spitzen Repositionszange und Einbringen der Kirschner-Drähte mit einer Parallelbohrbüchse. b Ausrichten von Kirschner-Drähten und Cerclagendraht. $\mathbf{c}$ und d Vervollständigte Zuggurtungsosteosynthese.

(Aus: Stöckle U, Hrsg. Ellenbogenchirurgie Standardverfahren, Tipps und Tricks. 2010. Mit freundlicher Genehmigung des Elsevier-Verlages, München.) 

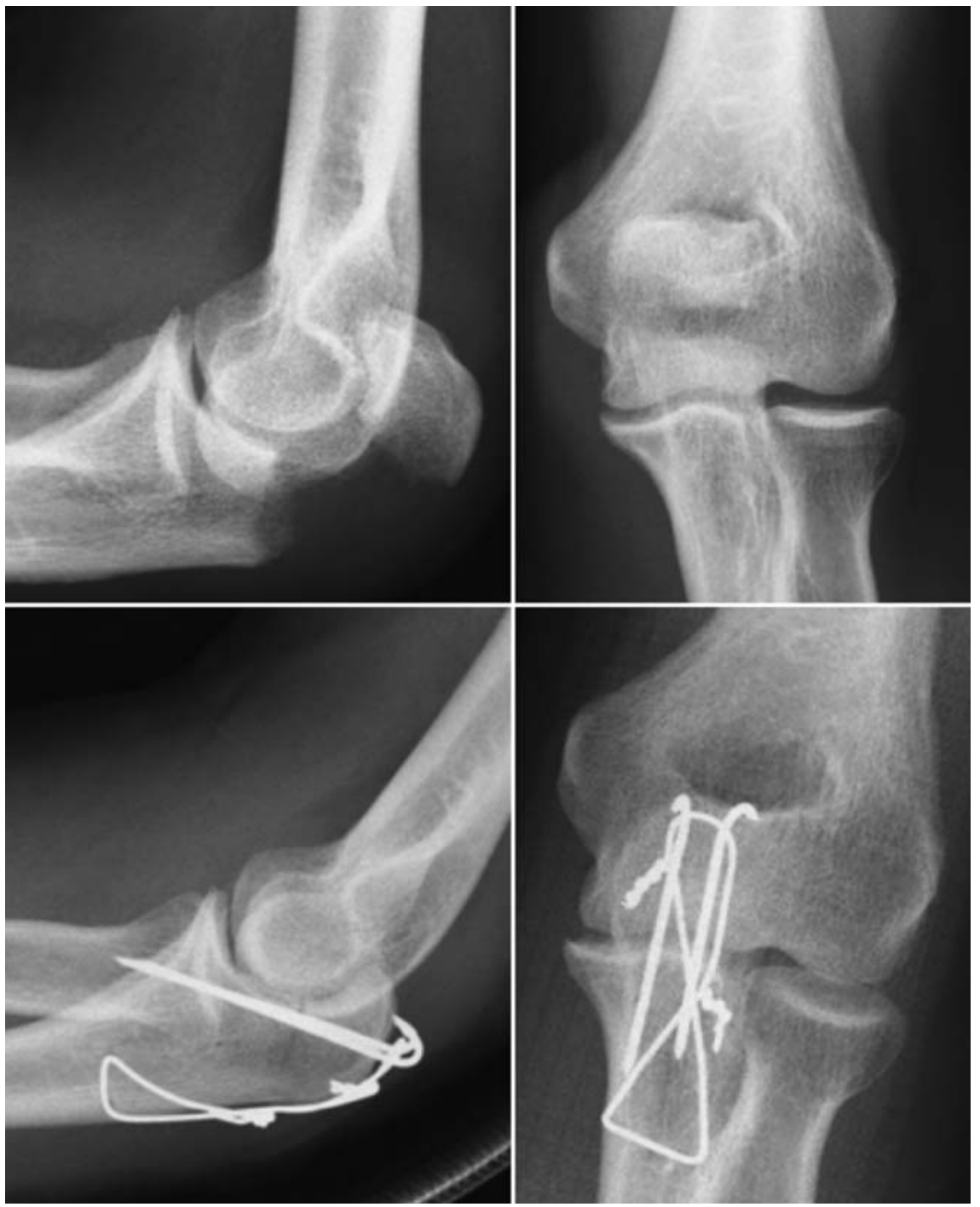

Abb. 4 Klinisches Beispiel eines 46-jährigen Patienten mit einfacher, quer verlaufender Zweifragmentfraktur des Olekranons. Oben Unfallbilder, unten postoperative Kontrolle nach Versorgung mit einer Zuggurtungsosteosynthese. (Aus: Stöckle U, Hrsg. Ellenbogenchirurgie Standardverfahren, Tipps und Tricks. 2010. Mit freundlicher Genehmigung des Elsevier-Verlages, München.)

Fixieren mit feinen Kirschner-Drähten hilfreich sein. Ebenso können Minifragmentschrauben für den Aufbau gelenkflächentragender Fragmente verwendet werden. Imprimierte Gelenkflächenanteile sollten behutsam angehoben und mit Spongiosa oder ggf. einem allogenen Knochenersatzmaterial unterfüttert werden. Beim Platzieren von temporär fixierenden Kirschner-Drähten sollte auf einen ausreichenden Abstand der Drähte zueinander geachtet werden, um das nachfolgende Ausrichten der Platte zwischen den Drähten nicht zu behindern.

Für das Anlegen der Platte wird der Trizepssehnenansatz im Faserverlauf gespalten, da das proximale Plattenende auf keinen Fall dem Sehnenansatz aufgelegt werden sollte.
Nach erfolgter Auswahl der Plattenlänge wird sie mit Schränkeisen entsprechend der Kontur der proximalen Ulna vorgebogen. Dabei sollte sich die Platte anatomisch anpassen, ohne im proximalen Anteil zu einem Impingement in der Fossa olecrani mit nachfolgendem Streckhindernis zu führen. Nach Überprüfung von korrekter Reposition und Plattenlage empfiehlt es sich, die Platte zunächst schaftseitig mit einer monokortikal eingebrachten Kortikalisschraube zu fixieren (Abb. 5). Als Nächstes wird eine lange Kortikalisschraube durch das proximale Plattenloch und das den Sehnenansatz tragende proximale Hauptfragment eingebracht. Beim Bohren empfiehlt es sich, die Ausrichtung des Bohrers unter dem Bildwandler im seitlichen Strahlengang zu kontrollieren. Die Schraube sollte das proximale Fragment möglichst zentral erfassen, nahe der Gelenkfläche durch den spongiösen Knochen verlaufen und sich distal des Proc. coronoideus in der ventralen Kortikalis verankern. Je nach Frakturmorphologie kann die Frakturzone kontrolliert unter Kompression gesetzt werden. Durch eine moderate Kompression lassen sich eingefügte Zwischenfragmente effektiv „verklemmen“ und somit stabil fixieren. Ein zuvor angehobenes Gelenkflächenimprimat sollte dagegen nur leicht unter Kompression gesetzt werden, um ein Zusammenschieben mit konsekutivem Versatz der Gelenkfläche zu verhindern. Durch den gelenknahen Verlauf der Schraube lässt sich aber auch hier eine gute zusätzliche Abstützung der Gelenkfläche erreichen. Ist eine weitere Kompression erwünscht, kann im nächsten Schritt die monokortikale Schraube ein wenig gelockert und das nächstfolgende, distal gelegene Plattenloch mit einer exzentrisch eingebrachten, bikortikal verankernden Schraube besetzt werden Abschließend erfolgt das Komplettieren der Montage: die Fixation der Platte erfolgt schaftseitig mit 3 bikortikal verankernden Schrauben. Das proximale Fragment wird mit einer weiteren Schraube gesichert. Der Ansatz der Trizepssehne wird mit einer Vicrylnaht über der Platte verschlossen. Durch die feste Verankerung der Platte am Schaft und die Zweipunktfixation der langen Schraube (proximales Plattenloch/Gegenkortikalis) wird bei dieser Form der Osteosynthese ein stabiler Rahmen geschaffen. Im Gegensatz zur Zuggurtungsosteosynthese wird durch diesen den Frakturbereich überbrückenden Rahmen eine weitere Kompression bei der frühfunktionellen Beübung des Ellenbogens im Frakturbereich verhindert (Abb. 6).

Mit den beiden geschilderten Osteosyntheseverfahren lassen sich die meisten Frakturen sicher und übungsstabil versorgen. Technische Probleme werden häufig durch ausgedehnte Trümmerzonen der Gelenkfläche und kleine, schwer zu fixierende Fragmente im Bereich der Olekranonspitze beobachtet. Besonders bei geriatrischen Patienten können ausgeprägt osteoporotische Knochenverhältnisse erschwerend hinzukommen. In solchen Fällen hat sich die Verwendung der anatomisch vorgeformten, der proximalen Ulna angepassten LCP Olekranon bewährt (Abb.7 und 8). Dieses Implantat bietet mehrere winkelstabile Verankerungsmöglichkeiten im proximalen Anteil des Olekranons und eignet 
Tab. 1

\begin{tabular}{|c|c|}
\hline Frakturform & $\begin{array}{l}\text { Osteosynthe- } \\
\text { severfahren }\end{array}$ \\
\hline $\begin{array}{l}\text { Zweifragmentfraktur } \\
\text { Frakturverlauf quer } \\
\text { gute Knochenqualität }\end{array}$ & $\begin{array}{l}\text { Zuggur- } \\
\text { tungsosteo- } \\
\text { synthese }\end{array}$ \\
\hline $\begin{array}{l}\text { Schrägfraktur } \\
\text { Querfraktur ventral des } \\
\text { Rotationszentrums } \\
\text { Fraktur des Proc. coro- } \\
\text { noideus } \\
\text { Mehrfragmentfraktur } \\
\text { Trümmerzone }\end{array}$ & $\begin{array}{l}\text { Plattenosteo- } \\
\text { synthese } \\
\text { LCDCP/LCP }\end{array}$ \\
\hline $\begin{array}{l}\text { Mehrfragment-/ } \\
\text { Trümmerfraktur } \\
\text { kleines proximales } \\
\text { Fragment } \\
\text { langstreckige Frakturzone } \\
\text { ausgeprägte Osteoporose }\end{array}$ & $\begin{array}{l}\text { LCP Olekra- } \\
\text { non }\end{array}$ \\
\hline $\begin{array}{l}\text { "Salvage“-Verfahren in } \\
\text { ausgesuchten Fällen }\end{array}$ & $\begin{array}{l}\text { Fragment- } \\
\text { exzision }\end{array}$ \\
\hline
\end{tabular}

sich daher insbesondere für osteoporotischen Knochen und mehrfragmentäre Situationen. Zusätzlich kann man eine Entlastung des Trizepssehnenzugs durch Anlage einer protektiven Zuggurtung mittels PDS-Kordel durch die Trizepssehne erreichen [3].

\section{Alternativen}

Liegen ausgedehnte Trümmersituationen bei schlechter Knochenqualität vor, so kann der proximale Anteil des Olekranons bei ansonsten stabilem Humeroulnargelenk auch reseziert werden. Die Trizepssehne wird anschließend gelenkflächennah refixiert. Bis zu 50\% der Gelenkfläche können laut Inhofe et al. [4], ohne eine verbleibende Gelenkinstabilität entfernt werden. Nachuntersuchungen haben bei Patienten nach Resektion im Vergleich zu osteosynthetisch versorgten Olekranonfrakturen keinen wesentlichen Kraftverlust ergeben [5]. Bei geriatrischen Patienten kann dieses Verfahren durchaus erwogen werden und ist in ausgesuchten Fällen sicherlich weniger komplikationsbelastet als eine Osteosynthese.

Ein anderes Verfahren bei ausgedehnter zentraler Zertrümmerung der Gelenkfläche wurde von Colton beschrieben [6]. Hierbei wird die Trümmerzone durch eine keilförmige, nach dorsal offene Osteotomie entfernt. Anschließend werden proximaler und distaler Gelenkanteil aneinandergefügt und mit einer Zuggurtungsosteosynthese stabilisiert. Der

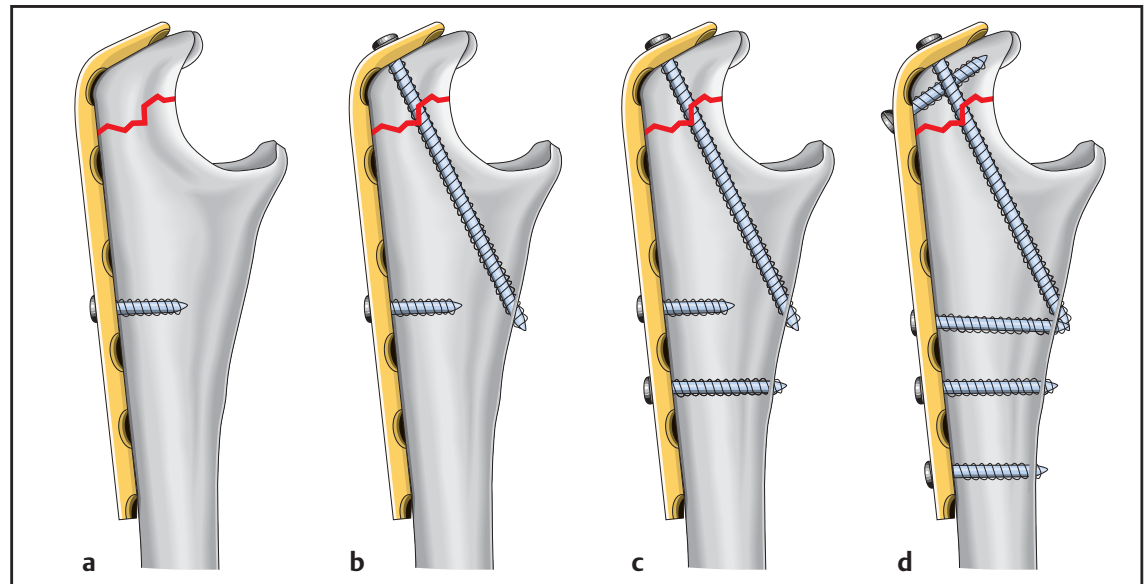

Abb. $\mathbf{5}$ a bis $\mathbf{d}$ Schematische Darstellung der Plattenosteosynthese mit einer KleinfragmentLCDCP (von links nach rechts). a Ausrichten der vorgeschränkten LCDCP und temporäre Fixation schaftseitig mit einer monokortikalen Kleinfragmentschraube. Die Platte sollte sich der proximalen Ulna harmonisch anfügen und die Olekranonspitze nicht überragen. b Platzieren der interfragmentären Schraube. Diese erfasst das proximale Fragment zentral, verläuft nahe der Gelenkfläche durch den spongiösen Knochen und verankert sich in der Gegenkortikalis ventral des Processus coronoideus. c Optional kann eine weitere Frakturkompression durch exzentrisches Einbringen einer weiteren Kleinfragmentschraube erfolgen. Während des Anziehens der exzentrisch eingebrachten Schraube wird die monokortikale Schraube gelockert. d Komplettieren der Montage. Die Fixation der Platte erfolgt schaftseitig mit 3 bikortikal verankernden Schrauben. Das proximale Fragment wird mit einer weiteren Schraube gesichert. (Aus: Stöckle U, Hrsg. Ellenbogenchirurgie Standardverfahren, Tipps und Tricks. 2010. Mit freundlicher Genehmigung des Elsevier-Verlages, München.)

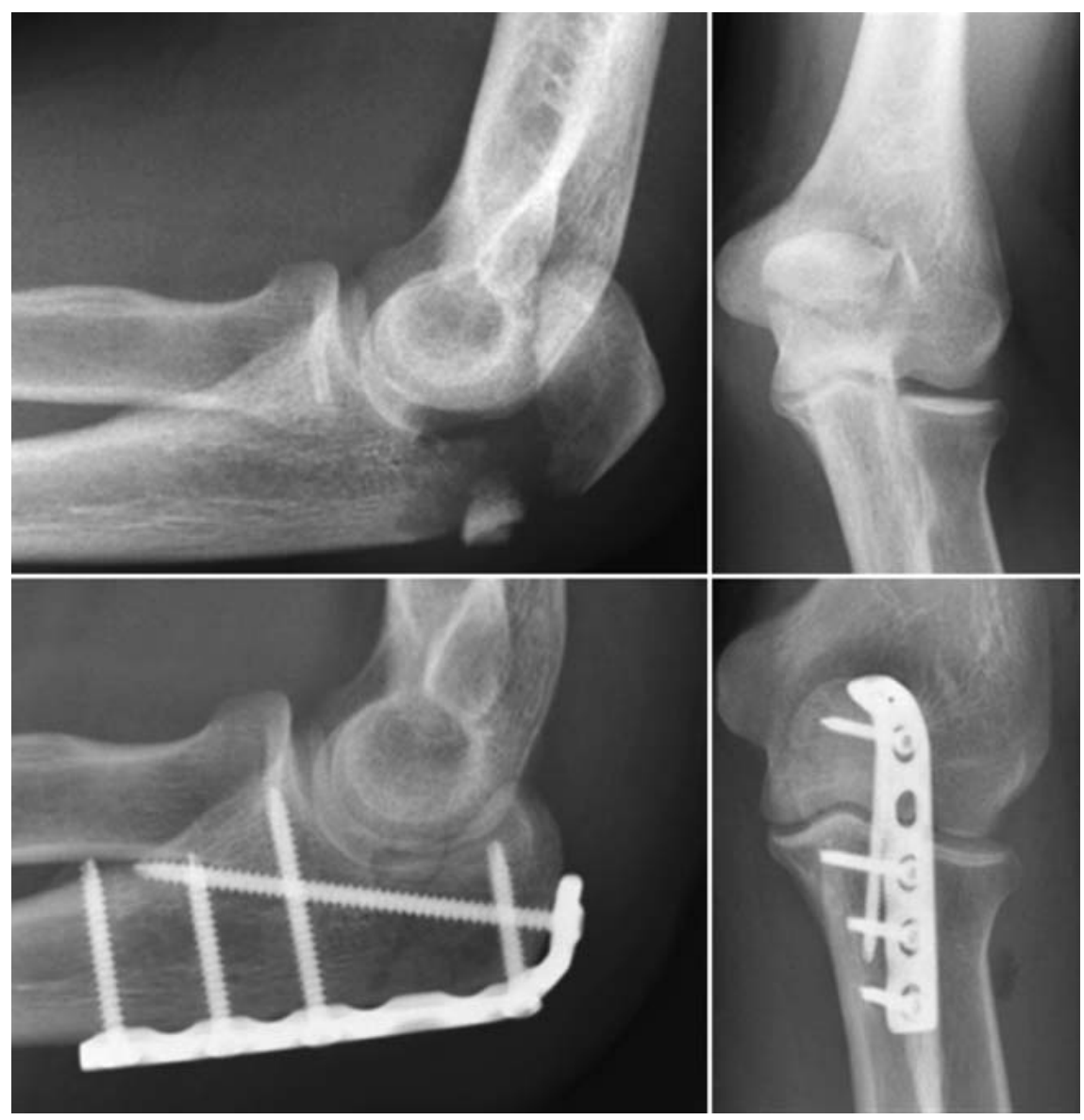

Abb. 6 Klinisches Beispiel eines 35-jährigen Patienten mit mehrfragmentärer Fraktur des Olekranons. Oben Unfallbilder, unten postoperative Kontrolle nach Versorgung durch Plattenosteosynthese mit einer LCDCP. (Aus: Stöckle U, Hrsg. Ellenbogenchirurgie Standardverfahren, Tipps und Tricks. 2010. Mit freundlicher Genehmigung des Elsevier-Verlages, München.) 

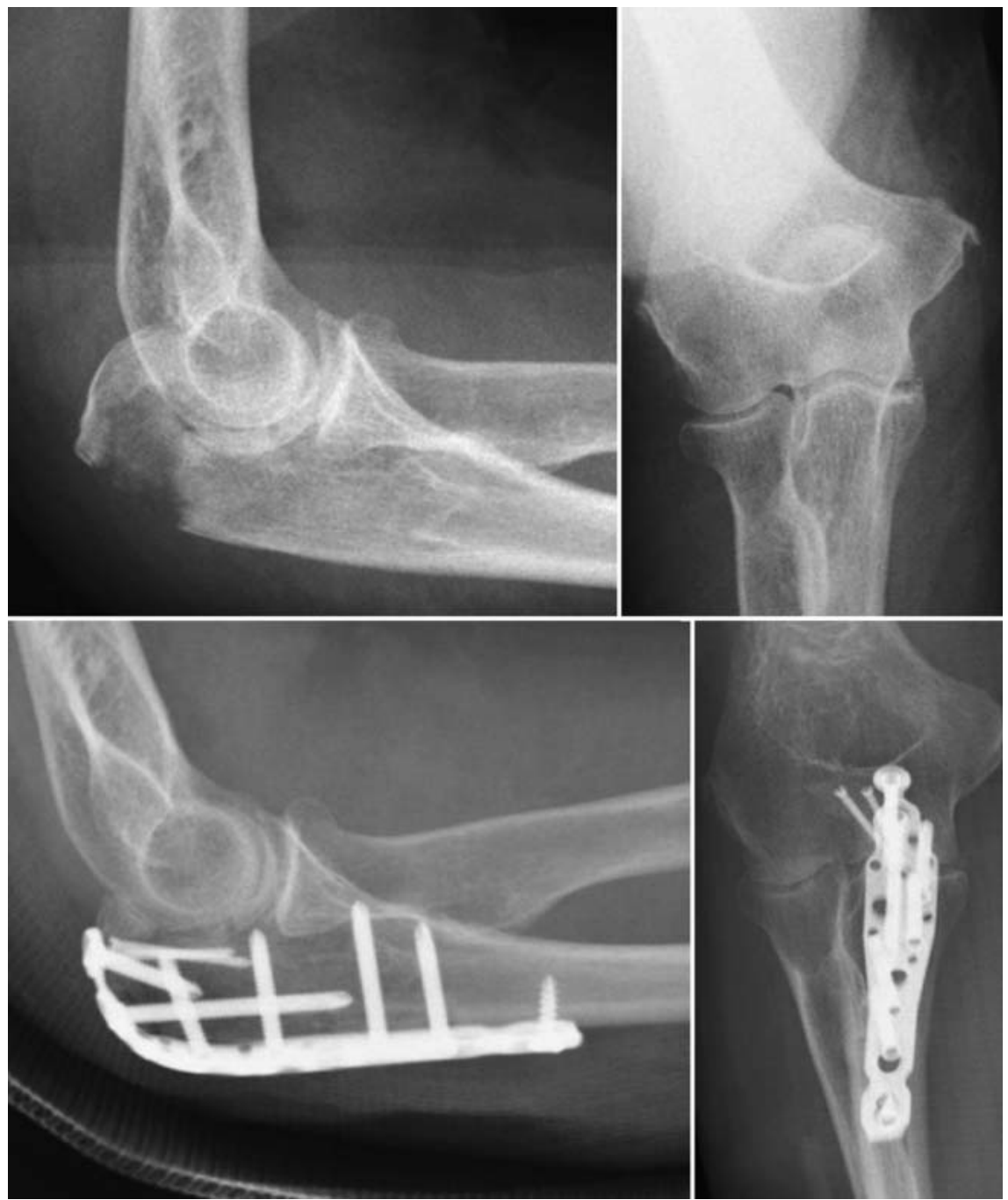

Abb. 7 Klinisches Beispiel einer 83-jährigen Patientin mit mehrfragmentärer Fraktur des Olekranons. Oben: Unfallbilder mit Gelenkflächenimpression und kleinem proximalem Fragment. Unten: postoperative Kontrolle nach Versorgung mit einer anatomisch vorgeformten winkelstabilen Olekranonplatte. Zusätzliche Fixation kleinerer Fragmente mit 2 Minischrauben. (Aus: Stöckle U, Hrsg. Ellenbogenchirurgie Standardverfahren, Tipps und Tricks. 2010. Mit freundlicher Genehmigung des Elsevier-Verlages, München.)

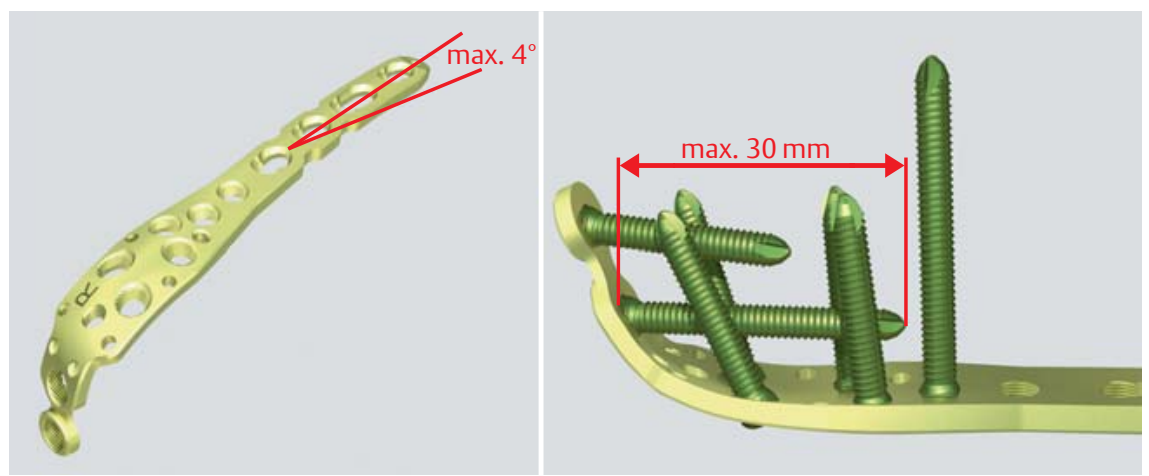

Abb. 8 Anatomisch vorgeformtes winkelstabiles Implantat zur Versorgung von Frakturen der proximalen Ulna (LCP Olekranon, Fa. Synthes). Das Implantatdesign berücksichtigt den in der Frontalebene leicht gebogenen Verlauf der proximalen Ulna. Für den gelenkflächentragenden Teil der proximalen Ulna stehen 7 winkelstabil verankerte Schraubenoptionen zur Verfügung. (Aus: Stöckle U, Hrsg. Ellenbogenchirurgie Standardverfahren, Tipps und Tricks. 2010. Mit freundlicher Genehmigung des Elsevier-Verlages, München.)
Winkel der Osteotomieebenen zueinander ist dabei so zu wählen, dass der Gelenkflächenradius nicht verändert wird.

Abschließend soll in Tab. 1 eine Übersicht der Kriterien im eigenen Vorgehen bei der Wahl des Osteosyntheseverfahrens gegeben werden.

\section{Komplikationen}

Häufige Komplikationen nach operativ versorgten Olekranonfrakturen sind schmerzhaft prominentes Osteosynthesematerial, meist verursacht durch Kirschner-Drähte nach Zuggurtungsosteosynthesen [7-9] oder Auslockerungen derselben mit konsekutiver Wanderung und Weichteilirritation bei insuffizienter Verankerung. Nach Plattenosteosynthesen werden schmerzhafte Weichteilirritationen deutlich seltener beschrieben $[10,11]$. Dennoch wird von der Mehrzahl der Patienten eine Besserung von Bewegungsausmaß und subjektiver Einschränkung nach Entfernung der Implantate berichtet [8].

\section{Nachbehandlung}

Es empfiehlt sich, nach erfolgter Osteosynthese intraoperativ eine Stabilitätsprüfung unter Bildwandlerkontrolle durchzuführen. Nach Entfernen der Wunddrainagen wird spätestens am 2 . postoperativen Tag mit der passiv geführten Gelenkbeübung begonnen. In der Regel begrenzen wir das Bewegungsausmaß bei mehrfragmentären Frakturen für 6 Wochen postoperativ symptomadaptiert auf Extension/Flexion $0^{\circ}-0^{\circ}-90^{\circ}$. Bei unkomplizierten Zuggurtungsosteosynthesen mit guter Knochenqualität kann symptomadaptiert auch progressiver beübt werden. Letztendlich richtet sich die Nachbehandlung jedoch nach der Stabilität der Osteosynthese und der Vorgabe des Operateurs.

\section{Ergebnisse}

Die operative Therapie isolierter dislozierter Olekranonfrakturen führt überwiegend zu guten bis sehr guten Ergebnissen [8-14].

In einer Veröffentlichung mit einem Follow-up von durchschnittlich 19 Jahren bei 73 Patienten mit einer isolierten Olekranonfraktur hatten $84 \%$ ein exzellentes Ergebnis mit keinerlei Beschwerden, $12 \%$ ein gutes mit gelegentlichen Schmerzen und $4 \%$ ein schlechtes mit täglichen Schmerzen. Der Bewegungsumfang war 
bei 38\% der Patienten mit dislozierter Zweifragmentfraktur und $43 \%$ der $\mathrm{Pa}$ tienten mit Mehrfragmentfraktur eingeschränkt. Radiologisch wurden degenerative Veränderungen in mehr als 50\% der Patienten gefunden. Eine manifeste Arthrose zeigte sich bei $6 \%$. Die radiologischen Veränderungen korrelierten nicht mit dem Ergebnis [14]. Rommens et al. [8] untersuchten retrospektiv 58 Patienten nach Osteosynthese einer Olekranonfraktur mit einem mittleren Follow-up von 3 Jahren. Offensichtlich scheinen dabei die Frakturmorphologie, die Primärstabilität des verletzten Ellenbogengelenks sowie die Qualität der Reposition und Osteosynthese wesentliche prognostische Faktoren hinsichtlich der zu erwartenden Funktion zu sein.

\section{Literatur}

${ }^{1}$ Hölzl A, Verheyden AP. Isolierte Olekranonfrakturen. Unfallchirurg 2008; 111: 727-734

${ }^{2}$ Bodler. Frakturen der proximalen Ulna aus Orthopädie und Unfallchirurgie (Grifka, Kusterer) Kapitel 24.2.5; 2011

${ }^{3}$ Lucke M. Olekranonfrakturen aus Ellenbogenchirurgie (Hrsg. Prof. U. Stöckle). Elsevier 2010

${ }^{4}$ Inhofe PD, Howard TC. The treatment of olecranon fractures by excision of fragments and repair of the extensor mechanism: historical review and report of 12 fractures. Orthopedics 1993; 16: 1313-1317

5 Gartsman GM, Sculco TP, Otis JC. Operative treatment of olecranon fractures. Excision or open reduction with internal fixation. J Bone Joint Surg [Am] 1981; 63: 718-721

${ }^{6}$ Colton CL. Fractures of the olecranon in adults. Injury 1973; 4: 121-129

7 Villanueva $P$, Osorio $F$, Commessatti $M$ et al. Tension-band wiring for olecranon fractures:analysis of risk factors for failure. J Shoulder Elbow Surg 2006; 15: 351-356

8 Rommens P, Küchle R, Schneider $R$ et al. Olecranon fractures in adults: factors influencing outcome. Injury 2004; 35: 1149-1157

${ }^{9}$ Romero JM, Miran A, Jensen CH. Complications and re-operation rate after tensionband wiring of olecranon fractures. J Orthop Sci 2000; 5: 318-320

10 Veillette CJH, Steinmann SP. Olecranon fractures. Orthop Clin North Am 2008; 39: 229236

11 Bailey CS, Mac Dermid J, Patterson SD et al. Outcome of plate fixation of olecranon fractures. J Orthop Trauma 2001; 15: 542-548

12 Lindenhovius AL, Brouwer KM, Doornberg JN et al. Long-term outcome of operatively treated fracture-dislocations of the olecranon. J Orthop Trauma 2008; 22: 325-331

13 Anderson ML, Larson AN, Merten SM et al. Congruent elbow plate fixation of olecranon fractures. J Orthop Trauma 2007; 21: 386393

${ }^{14}$ Karlsson MK, Hasserius R, Karlsson $C$ et al. Fractures of the olecranon: a 15- to 25-year followup of 73 patients. Clin Orthop Relat Res 2002; 403: 205-212
15 Charlton WP, Chandler RW. Persistence of the olecranon physis in baseball players: results following operative management. J Shoulder Elbow Surg 2003; 12: 59-62

16 Weigel B, Nerlich ML. Praxisbuch Unfallchirurgie $2011 ; 6$ : 346-350

Dr. med. Manuel Baacke

Assistenzarzt

Dr. med. Atesch Ateschrang Oberarzt

Univ.-Prof. Dr. Ulrich Stöckle

Ärztlicher Direktor

Klinik für Unfall- und

Wiederherstellungschirurgie

BGU Klinik Tübingen

Schnarrenbergstraße 95

72076 Tübingen

ustoeckle@bgu-tuebingen.de

Dr. med. Martin Lucke

Leitender Oberarzt

Klinik für Unfallchirurgie

Klinikum rechts der Isar

Ismaningerstraße 22

81675 München 